\title{
Cumulative Author Index
}

\section{Volume 6 (2017)}

Adami, K. Z., see Naldi, G.

Adami, K. Z., see Comoretto, G.

Ajith Kumar, B., see Reddy, S. H.

Alderighi, M., see Naldi, G.

Alderighi, M., see Comoretto, G.

Allison, P., see Gorham, P. W. Allison, P., see Baginski, F.

Ambily, S., Sarpotdar, M., Mathew, J., Sreejith, A. G., Nirmal, K., Prakash, A., Safonova, M. and Murthy, J., Development of Data Acquisition Methods for an FPGA-Based Photon Counting Detector

Aminaei, A., see Naldi, G.

Aminaei, A., see Comoretto, G. Anderson, C. J., see Cianciara, A. J.

Andrianov, A. S., see Likhachev, S. F.

Anupam, G. C., see Sharma, T. K.

Armstrong, R., see Van Belle, J. Baginski, F., Zhao, K., Furer, J., Landay, J., Bailoor, S., Gorham, P., Varner, G., Miki, C., Hill, B., Schoorlemmer, H., Nguyen, L., Romero-Wolf, A., Liewer, K., Sauder, J., Brakke, K., Beatty, J., Connolly, A., Allison, P., Pfendner, C., Dailey, B., Fairbrother, D., Said, M., Lang, S. and Young, L., Shape Analysis and Deployment of the ExaVolt Antenna
1 (2017) 1641014

1 (2017) 1641015

1 (2017) 1641011

1 (2017) 1641014

1 (2017) 1641015

2 (2017) 1740002

2 (2017) 1740004

1 (2017) 1750002

1 (2017) 1641014

1 (2017) 1641015

3 (2017) 1750003

3 (2017) 1750004

4 (2017) 1750008

4 (2017) 1750009

2 (2017) 1740004
Bailoor, S., see Baginski, F. Baker, J., see Naldi, G.

2 (2017) 1740004

1 (2017) 1641014

Baker, J., see Comoretto, G.

Balasubramaniam, K. A., see Deshmukh, P. G.

1 (2017) 1641015

3 (2017) 1750006

Banerjee, O., see Gorham, P. W.

2 (2017) 1740002

Banyal, R. K. and Reiners, A., A Dual Cavity Fabry-Perot Device for High Precision Doppler Measurements in Astronomy

Banyal, R. K., see Sharma, T. K.

Barsdell, B. R., see Cranmer, M. D.

Beatty, J. J., see Gorham, P. W.

1 (2017) 1750001

4 (2017) 1750008

4 (2017) 1750007

Beatty, J., see Baginski, F.

Beheshtipour, B., see Kislat, F.

Belli, C., see Naldi, G.

Belli, C., see Comoretto, G.

Belov, K., see Gorham, P. W.

Besson, D. Z., see Gorham, P. W.

Binns, W. R., see Gorham, P. W.

2 (2017) 1740002

2 (2017) 1740004

2 (2017) 1740003

1 (2017) 1641014

1 (2017) 1641015

2 (2017) 1740002

2 (2017) 1740002

2 (2017) 1740002

2 (2017) 1740004

2 (2017) 1740003

2 (2017) 1740002

2 (2017) 1740003

2 (2017) 1740002

2 (2017) 1740007

Card, G., see Lecinski, A.

Chaudhari, S. C., Gupta, Y., Kumar, A., Shinde, N. D., Gupta, S. and Vishwakarma, A., Reducing Effects of CrossTalk in a Radio Telescope Using Walsh Modulation

1 (2017) 1641017

Chengalur, J. N., Interferometry and Synthesis in Radio Astronomy 
Chen, C., see Gorham, P. W. Chen, P., see Gorham, P. W. Chen, X., see Cianciara, A. J. Chen, Z., see Cianciara, A. J. Chiarucci, S., see Naldi, G.

Chiarucci, S., see Comoretto, G. Chiello, R., see Naldi, G.

Chiello, R., see Comoretto, G. Cianciara, A. J., Anderson, C. J., Chen, X., Chen, Z., Geng, J., Li, J., Liu, C., Liu, T., Lu, W., Peterson, J. B., Shi, H., Steffel, C. N., Stebbins, A., Stucky, T., Sun, S., Timbie, P. T., Wang, Y., Wu, F. and Zhang, J., Simulation and Testing of a Linear Array of Modified Four-Square Feed Antennas for the Tianlai Cylindrical Radio Telescope Clem, J. M., see Gorham, P. W. Comoretto, G., see Naldi, G.

Comoretto, G., Chiello, R., Roberts, M., Halsall, R., Adami, K. Z., Alderighi, M., Aminaei, A., Baker, J., Belli, C., Chiarucci, S., D'Angelo, S., De Marco, A., Mura, G. D., Magro, A., Mattana, A., Monari, J., Naldi, G., Pastore, S., Perini, F., Poloni, M., Pupillo, G., Rusticelli, S., Schiaffino, M., Schillirò, F. and Zaccaro, E., The Signal Processing Firmware for the Low Frequency Aperture Array

Connolly, A., see Gorham, P. W.

Connolly, A., see Baginski, F. Cranmer, M. D., Barsdell, B. R., Price, D. C., Dowell, J., Garsden, H., Dike, V., Eftekhari, T., Hegedus, A. M., Malins, J., Obenberger, K. S., Schinzel, F., Stovall, K., Taylor, G. B. and Greenhill, L. J., Bifrost: A Python/C++ Framework for High-Throughput Stream Processing in Astronomy
2 (2017) 1740002

2 (2017) 1740002

3 (2017) 1750003

3 (2017) 1750003

1 (2017) 1641014

1 (2017) 1641015

1 (2017) 1641014

1 (2017) 1641015

3 (2017) 1750003

2 (2017) 1740002

1 (2017) 1641014

1 (2017) 1641015

2 (2017) 1740002

2 (2017) 1740004

4 (2017) 1750007
Cremonesi, L., see Gorham, P. W.

2 (2017) 1740002

Dailey, B., see Gorham, P. W. 2 (2017) 1740002

Dailey, B., see Baginski, F.

D'Angelo, S., see Naldi, G.

D'Angelo, S., see Comoretto, G.

Dasgupta, P., see Gorham, P. W.

2 (2017) 1740004

1 (2017) 1641014

1 (2017) 1641015

2 (2017) 1740002

1 (2017) 1641011

2 (2017) 1740003

1 (2017) 1641014

De Marco, A., see Naldi, G.

De Marco, A., see Comoretto, G.

Deaconu, C., see Gorham, P. W.

Deshmukh, P. G., Parihar, P., Balasubramaniam, K. A., Mishra, D. S. and Mahesh, P. K., Dynamic Loading Assembly for Testing Actuators of Segmented Mirror Telescope

1 (2017) 1641015

2 (2017) 1740002

Devlin, M. J., see Nati, F.

Dike, V., see Cranmer, M. D.

Dowell, J., see Cranmer, M. D.

Dowkontt, P., see Kislat, F.

Dowkontt, P. F., see Gorham, P. W.

Eftekhari, T., see Cranmer, M. D.

Fairbrother, D., see Baginski, $\mathrm{F}$.

Fox, B. D., see Gorham, P. W.

Fuke, H., Recent Highlights of Scientific Ballooning in Japan

Fuke, H., see Shoji, Y.

Fuke, H., Okazaki, S., Ogawa, H. and Miyazaki, Y., Balloon Flight Demonstration of an Oscillating Heat Pipe

Furer, J., see Baginski, F.

Gain, J., see Van Belle, J.

Gamatham, R. R. G., see

Rotich Kipnoo, E. K.

Garsden, H., see Cranmer, M. D.

Geng, J., see Cianciara, A. J.

Gerbino, M., see Nati, F.

Gibbon, T. B., see Rotich

Kipnoo, E. K.
3 (2017) 1750006

2 (2017) 1740008

4 (2017) 1750007

4 (2017) 1750007

2 (2017) 1740003

2 (2017) 1740002

4 (2017) 1750007

2 (2017) 1740004

2 (2017) 1740002

2 (2017) 1740001

2 (2017) 1740005

2 (2017) 1740006

2 (2017) 1740004

4 (2017) 1750009

3 (2017) 1750005

4 (2017) 1750007

3 (2017) 1750003

2 (2017) 1740008

3 (2017) 1750005 
Girin, I. A., see Likhachev, S. F.

Gnanaraj, S., see Reddy, S. H.

Gokhale, U., see Reddy, S. H. Gordon, J., see Gorham, P. W. Gorham, P., see Baginski, F.

Gorham, P. W., Allison, P., Banerjee, O., Beatty, J. J., Belov, K., Besson, D. Z., Binns, W. R., Bugaev, V., Cao, P., Chen, C., Chen, P., Clem, J. M., Connolly, A., Dailey, B., Dasgupta, P., Deaconu, C., Cremonesi, L., Dowkontt, P. F., Fox, B. D., Gordon, J., Hill, B., Hupe, R., Israel, M. H., Jain, P., Kowalski, J., Lam, J., Learned, J. G., Liewer, K. M., Liu, T. C., Matsuno, S., Miki, C., Mottram, M., Mulrey, K., Nam, J., Nichol, R. J., Novikov, A., Oberla, E., Prohira, S., Rauch, B. F., Romero-Wolf, A., Rotter, B., Ratzlaff, K., Russell, J., Saltzberg, D., Seckel, D., Schoorlemmer, H., Stafford, S., Stockham, J., Stockham, M., Strutt, B., Tatem, K., Varner, G. S., Vieregg, A. G., Wissel, S. A., Wu, F. and Young, R., Antarctic Surface Reflectivity Measurements from the ANITA-3 and HiCal-1 Experiments

Greenhill, L. J., see Cranmer, M. D.

Guarino, V., see Kislat, F.

Gupta, S., see Chaudhari, S. C. Gupta, Y., see Reddy, S. H.

Gupta, Y., see Chaudhari, S. C.

Halagalli, I., see Reddy, S. H. Halsall, R., see Naldi, G.

Halsall, R., see Comoretto, G. Hamada, K., see Shoji, Y.

Hardy, B., see Lecinski, A. Heatwole, S., see Kislat, F.
3 (2017) 1750004

1 (2017) 1641011

1 (2017) 1641011

2 (2017) 1740002

2 (2017) 1740004

2 (2017) 1740002

4 (2017) 1750007

2 (2017) 1740003

1 (2017) 1641017

1 (2017) 1641011

1 (2017) 1641017

1 (2017) 1641011

1 (2017) 1641014

1 (2017) 1641015

2 (2017) 1740005

2 (2017) 1740007

2 (2017) 1740003
Hegedus, A. M., see Cranmer, M. D.

4 (2017) 1750007

Hill, B., see Gorham, P. W.

Hill, B., see Baginski, F.

Hoorman, J., see Kislat, F.

Hupe, R., see Gorham, P. W.

Iijima, I., see Shoji, Y.

Ikeda, C., see Shoji, Y.

Israel, M. H., see Gorham, P. W.

Iyer, B., An Overview of Gravitational Waves: Theory,

Sources and Detection

Izutsu, N., see Shoji, Y.

Jain, P., see Gorham, P. W.

Johnson, B. R., see Nati, F.

Kakehashi, Y., see Shoji, Y.

Keating, B., see Nati, F.

Kemkar, M. M., see Sharma, T. K.

Kirsten, T., Neutrino Astronomy: Current Status, Future Prospects

2 (2017) 1740002

2 (2017) 1740004

2 (2017) 1740003

2 (2017) 1740002

2 (2017) 1740005

2 (2017) 1740005

2 (2017) 1740002

3 (2017) 1780001

2 (2017) 1740005

2 (2017) 1740002

2 (2017) 1740008

2 (2017) 1740005

2 (2017) 1740008

4 (2017) 1750008

Kislat, F., Beheshtipour, B., Dowkontt, P., Guarino, V., Lanzi, R. J., Okajima, T., Braun, D., Cannon, S., De Geronimo, G., Heatwole, S., Hoorman, J., Li, S., Mori, H., Shreves, C. M., Stuchlik, D. and Krawczynski, H., Design of the Telescope Truss and Gondola for the BalloonBorne X-Ray Polarimeter $X$-Calibur

2 (2017) 1740003

Knölker, M., see Lecinski, A.

Kostenko, V. I., see Likhachev, S. F.

Kowalski, J., see Gorham, P. W.

4 (2017) 1780004

3 (2017) 1750004
Krawczynski, H., see Kislat, F. 2 (2017) 1740003

Kudale, S., see Reddy, S. H. $\quad 1$ (2017) 1641011

Kumar, A., see Chaudhari, S. C.

1 (2017) 1641017

Lam, J., see Gorham, P. W. $\quad 2$ (2017) 1740002

Landay, J., see Baginski, F. 2 (2017) 1740004

Lang, S., see Baginski, F. $\quad 2$ (2017) 1740004

Lanzi, R. J., see Kislat, F. $\quad 2$ (2017) 1740003

Learned, J. G., see Gorham, P. W.

2 (2017) 1740002 
Lecinski, A., Card, G., Knölker, M. and Hardy, B., The Design and Performance of the Gondola Pointing System for the Sunrise II Balloon-Borne Stratospheric Solar Observatory

Leitch, A. W. R., see Rotich Kipnoo, E. K.

Liewer, K., see Baginski, F.

Liewer, K. M., see Gorham, P. W.

Li, J., see Cianciara, A. J.

Li, S., see Kislat, F.

Likhachev, S. F., Kostenko, V. I., Girin, I. A., Andrianov, A. S., Rudnitskiy, A. G. and Zharov, V. E., Software Correlator for Radioastron Mission

Liu, C., see Cianciara, A. J.

Liu, T., see Cianciara, A. J.

Liu, T. C., see Gorham, P. W.

Lu, W., see Cianciara, A. J.

Magro, A., see Naldi, G.

Magro, A., see Comoretto, G.

Mahesh, P. K., see Deshmukh, P. G.

Malins, J., see Cranmer, M. D. Mathew, J., see Ambily, S.

Matsuno, S., see Gorham, P. W.

Matsuzaka, Y., see Shoji, Y. Mattana, A., see Naldi, G.

Mattana, A., see Comoretto, G. Miki, C., see Gorham, P. W.

Miki, C., see Baginski, F.

Mishra, D. S., see Deshmukh, P. G.

Miyazaki, Y., see Fuke, H.

Monari, J., see Naldi, G.

Monari, J., see Comoretto, G.

Mori, H., see Kislat, F.

Mottram, M., see Gorham, P. W.

Mulrey, K., see Gorham, P. W.

Mura, G. D., see Naldi, G.

Mura, G. D., see Comoretto, G.

Murthy, J., see Ambily, S.

Naldi, G., see Comoretto, G.

\section{2 (2017) 1740007}

3 (2017) 1750005

2 (2017) 1740004

2 (2017) 1740002

3 (2017) 1750003

2 (2017) 1740003

3 (2017) 1750004

3 (2017) 1750003

3 (2017) 1750003

2 (2017) 1740002

3 (2017) 1750003

1 (2017) 1641014

1 (2017) 1641015

3 (2017) 1750006

4 (2017) 1750007

1 (2017) 1750002

2 (2017) 1740002

2 (2017) 1740005

1 (2017) 1641014

1 (2017) 1641015

2 (2017) 1740002

2 (2017) 1740004

3 (2017) 1750006

2 (2017) 1740006

1 (2017) 1641014

1 (2017) 1641015

2 (2017) 1740003

2 (2017) 1740002

2 (2017) 1740002

1 (2017) 1641014

1 (2017) 1641015

1 (2017) 1750002

1 (2017) 1641015
Naldi, G., Mattana, A., Pastore, S., Alderighi, M., Adami, K. Z., Schillirò, F., Aminaei, A., Baker, J., Belli, C., Comoretto, G., Chiarucci, S., Chiello, R., D'Angelo, S., Mura, G. D., De Marco, A., Halsall, R., Magro, A., Monari, J., Roberts, M., Perini, F., Poloni, M., Pupillo, G., Rusticelli, S., Schiaffino, M. and Zaccaro, E., The Digital Signal Processing Platform for the Low Frequency Aperture Array: Preliminary Results on the Data Acquisition Unit Nam, J., see Gorham, P. W. Nati, F., Devlin, M. J., Gerbino, M., Johnson, B. R., Keating, B., Pagano, L. and Teply, G., POLOCALC: A Novel Method to Measure the Absolute Polarization Orientation of the Cosmic Microwave Background

Nguyen, L., see Baginski, F.

Nichol, R. J., see Gorham, P. W.

Nirmal, K., see Ambily, S.

Novikov, A., see Gorham, P. W.

Obenberger, K. S., see Cranmer, M. D.

1 (2017) 1641014

2 (2017) 1740002

2 (2017) 1740008

2 (2017) 1740004

2 (2017) 1740002

1 (2017) 1750002

2 (2017) 1740002

Oberla, E., see Gorham, P. W.

Ogawa, H., see Fuke, H.

Okajima, T., see Kislat, F.

Okazaki, S., see Fuke, H.

Pagano, L., see Nati, F.

Parihar, P., see Deshmukh, P. G.

Parihar, P., see Sharma, T. K.

Pastore, S., see Naldi, G.

Pastore, S., see Comoretto, G.

Perini, F., see Naldi, G.

Perini, F., see Comoretto, G.

Peterson, J. B., see Cianciara, A. J.

Pfendner, C., see Baginski, F.

Pollacco, D., Methods of Detecting Exoplanets

Poloni, M., see Naldi, G.
4 (2017) 1750007

2 (2017) 1740002

2 (2017) 1740006

2 (2017) 1740003

2 (2017) 1740006

2 (2017) 1740008

3 (2017) 1750006

4 (2017) 1750008

1 (2017) 1641014

1 (2017) 1641015

1 (2017) 1641014

1 (2017) 1641015

3 (2017) 1750003

2 (2017) 1740004

4 (2017) 1780003

1 (2017) 1641014 
Poloni, M., see Comoretto, G.

Prakash, A., see Ambily, S.

Price, D. C., see Cranmer, M. D.

Prohira, S., see Gorham, P. W.

Pupillo, G., see Naldi, G.

Pupillo, G., see Comoretto, G.

Raskar, N., see Reddy, S. H.

Ratzlaff, K., see Gorham, P. W.

Rauch, B. F., see Gorham, P. W.

Reddy, S. H., Kudale, S., Gokhale, U., Halagalli, I., Raskar, N., De, K., Gnanaraj, S., Ajith Kumar, B. and Gupta, Y., A Wideband Digital Back-End for the Upgraded GMRT

Reiners, A., see Banyal, R. K.

Roberts, M., see Naldi, G.

Roberts, M., see Comoretto, G.

Romero-Wolf, A., see Gorham, P. W.

Romero-Wolf, A., see Baginski, F.

Rotich Kipnoo, E. K., Gamatham, R. R. G., Leitch, A. W. R. and Gibbon, T. B., Simultaneous Signal Amplification and Clock Distribution Employing Backward Raman Pump Over an Optical Fiber for Applications Such as Square Kilometer Array

Rotter, B., see Gorham, P. W.

Rudnitskiy, A. G., see

Likhachev, S. F.

Russell, J., see Gorham, P. W.

Rusticelli, S., see Naldi, G.

Rusticelli, S., see Comoretto, G.

Safonova, M., see Ambily, S.

Said, M., see Baginski, F.

Saltzberg, D., see Gorham, P. W.

Sarpotdar, M., see Ambily, S.

Sato, T., see Shoji, Y.

Sauder, J., see Baginski, F.

Schiaffino, M., see Naldi, G.

Schiaffino, M., see Comoretto, G.
1 (2017) 1641015

1 (2017) 1750002

4 (2017) 1750007

2 (2017) 1740002

1 (2017) 1641014

1 (2017) 1641015

1 (2017) 1641011

2 (2017) 1740002

2 (2017) 1740002

1 (2017) 1641011

1 (2017) 1750001

1 (2017) 1641014

1 (2017) 1641015

2 (2017) 1740002

2 (2017) 1740004

3 (2017) 1750005

2 (2017) 1740002

3 (2017) 1750004

2 (2017) 1740002

1 (2017) 1641014

1 (2017) 1641015

1 (2017) 1750002

2 (2017) 1740004

2 (2017) 1740002

1 (2017) 1750002

2 (2017) 1740005

2 (2017) 1740004

1 (2017) 1641014

1 (2017) 1641015
Schillirò, F., see Naldi, G.

1 (2017) 1641014

Schillirò, F., see Comoretto, G.

Schinzel, F., see Cranmer, M. D.

Schoorlemmer, H., see

Gorham, P. W.

1 (2017) 1641015

4 (2017) 1750007

Schoorlemmer,

H., see

Baginski, F.

2 (2017) 1740002

Seckel, D., see Gorham, P. W.

Sharma, T. K., Parihar, P.,

Banyal, R. K., Kemkar, M.

M., Stanzin, U. and Anupam,

G. C., Infrared Scanning

Cloud Monitor for the Indian

Astronomical Observatory,

Hanle

Shi, H., see Cianciara, A. J.

Shinde, N. D., see Chaudhari,

S. C.

Shoji, Y., Fuke, H., Hamada,

K., Iijima, I., Ikeda, C.,

Izutsu, N., Kakehashi, Y.,

Matsuzaka, Y., Sato, T.,

Tamura, M. and Yoshida,

T., Marine Search-and-

Recovery Operation of

Scientific Balloons in Japan

Shreves, C. M., see Kislat, F.

Sreejith, A. G., see Ambily, S.

Stafford, S., see Gorham, P. W.

Stanzin, U., see Sharma, T. K.

Stebbins, A., see Cianciara,

A. J.

Steffel, C. N., see Cianciara,

A. J.

Stockham, J., see Gorham, P. W.

Stockham, M., see Gorham, P. W.

Stovall, K., see Cranmer, M. D. Strutt, B., see Gorham, P. W. Stuchlik, D., see Kislat, F.

Stucky, T., see Cianciara, A. J. Sun, S., see Cianciara, A. J.

Tamura, M., see Shoji, Y.

4 (2017) 1750008

3 (2017) 1750003

1 (2017) 1641017

2 (2017) 1740005

2 (2017) 1740003

1 (2017) 1750002

2 (2017) 1740002

4 (2017) 1750008

3 (2017) 1750003

3 (2017) 1750003

2 (2017) 1740002

2 (2017) 1740002

4 (2017) 1750007

2 (2017) 1740002

2 (2017) 1740003

3 (2017) 1750003

3 (2017) 1750003

2 (2017) 1740005

Tatem, K., see Gorham, P. W. 2 (2017) 1740002

Taylor, G. B., see Cranmer, M. D.

4 (2017) 1750007

2 (2017) 1740008

Timbie, P. T., see Cianciara,

A. J.

3 (2017) 1750003 
Van Belle, J., Armstrong, R. and Gain, J., Accelerated Deconvolution of Radio Interferometric Images using Orthogonal Matching Pursuit and Graphics Hardware

Varner, G., see Baginski, F.

Varner, G. S., see Gorham, P. W.

Vieregg, A. G., see Gorham, P. W.

Vishwakarma, A., see
Chaudhari, S. C.

Wang, Y., see Cianciara, A. J.
Wissel, S. A., see Gorham, P. W.

2 (2017) 1740002

Wu, F., see Gorham, P. W. $\quad 2$ (2017) 1740002

Wu, F., see Cianciara, A. J. 3 (2017) 1750003

Yoshida, T., see Shoji, Y. $\quad 2$ (2017) 1740005

4 (2017) 1750009

Young, L., see Baginski, F.

2 (2017) 1740004

Young, R., see Gorham, P. W. 2 (2017) 1740002

Zaccaro, E., see Naldi, G. $\quad 1$ (2017) 1641014

$2(2017) 1740002$

Zaccaro, E., see Comoretto, G.

Zhang, J., see Cianciara, A. J.

Zhao, K., see Baginski, F.

Zharov, V. E., see Likhachev,

1 (2017) 1641017

S. F.
1 (2017) 1641015

3 (2017) 1750003

2 (2017) 1740004

3 (2017) 1750004 